\title{
CANAL EXPERIMENTAL PARA ELIMINACIÓN DE HIERRO Y ARSÉNICO EN EL AGUA DEL RÍO TARUCACHI
}

Leoncio Molina Vásquez ${ }^{\prime}$ René Álvarez Pacheco ${ }^{2}$

\author{
RES U M E N
}

El proyecto de investigación tecnológico está basado en el módulo del canal experimental construido en la quebrada de Kejane, donde con el caudal medido, análisis químico, interacción de la piedra caliza con el agua del rio Tarucachi, Tarata - Perú, se calcula la longitud del canal experimental, número de filtros para incrementar el $\mathrm{pH}$ de 2,48 hasta hacerla apta para uso agropecuario, eliminando la acidez, hierro y arsénico que la contaminan, como una primera aproximación.

\section{A B S TR A C T}

The technological research project is based on the model of the experimental channel built in the Kejane gorge, where there is measured flow, chemical analysis, and interaction of the limestone with the water of the river Tarucachi, Tarata-Perú. The length of the experimental channel is calculated, as well as the number of filters required to increase the $\mathrm{pH}$ from 2.48 to a level suitable for farming use, eliminating acidity, iron and arsenic wich contaminate it as a first approximation. 


\section{INTRODUCCIÓN}

El trabajo se basa en el proyecto de investigación científica Interacción y Eliminación de Arsénico y Hierro del río Tarucachi.

El trabajo se propone encontrar una tecnologia adecuada para el tratamiento de las aguas del río Tarucachi y hacerla apta para uso agropecuario; rehabilitando así 800 hectáreas de uso agrícola.

\section{FUNDAMENTO CIENTÍFICO}

\subsection{CONCENTRACIÓN DE CONTAMINANTES}

Los procesos físico-químicos que ocurren durante la meteorización son complejos y variados, porque en el rio Tarucachi existen muchos sólidos disueltos en el agua y fundamentalmente se encuentran como iones: $\mathrm{Na}^{+}, \mathrm{K}^{+}, \mathrm{Fe}^{3}+, \mathrm{As}^{5+}, \mathrm{Ca}^{2+}, \mathrm{Cl}, \mathrm{SO}_{4}{ }^{2}, \mathrm{HCO}_{3} \cdot \mathrm{H}^{+} \mathrm{y}$ suspensiones coloidales de $\mathrm{Fe}(\mathrm{OH})_{3}$

En las aguas superficiales se oxidan incluso los minerales metálicos, produciendo soluciones que disuelven a su vez, a otros minerales; en este caso, y de acuerdo con la investigación geológica y litológica, se ha determinado que las aguas geotermales pasan por zonas mineralizadas de pirita arsenical y este mineral al ser tratado en caliente da fácilmente $\mathrm{Fe} \mathrm{SO}_{4}$ y $\mathrm{H}_{2} \mathrm{SO}_{4}$, como se ve en las reacciones.

$$
\begin{aligned}
& \mathrm{S}_{2} \mathrm{Fe}+7 / 2 \mathrm{O}_{2}+\mathrm{H}_{2} \mathrm{O} \longrightarrow \mathrm{Fe} \mathrm{SO} \mathrm{H}_{4}+\mathrm{H}_{2} \mathrm{SO}_{4} \\
& 2 \mathrm{Fe} \mathrm{SO}_{4}+\mathrm{H}_{2} \mathrm{SO}_{4}+1 / 2 \mathrm{O}_{2} \longrightarrow \mathrm{Fe}_{2}\left(\mathrm{SO}_{4}\right)_{3}+\mathrm{H}_{2} \mathrm{O} \\
& \mathrm{H}_{2} \mathrm{SO}_{4} \longrightarrow 2 \mathrm{H}^{+}+\mathrm{SO}^{2 \cdot 4}
\end{aligned}
$$

Estas reacciones dan el carácter ácido prónunciado que se expresa en el pH; los otros componentes que se ven en las tablas Nos. 1 - 2, deben tener igual origen. Tal es el caso del arsénico que debe originarse en la arsenopirita. La fórmula del río Tarucachi, de acuerdo a lo establecido por GASTANY y a la Tabla No. 2 es : $\mathrm{SO}_{4}{ }^{2 \cdot}, \mathrm{CO}_{3}{ }^{2-}, \mathrm{Cl}, \mathrm{Ca}^{2+}, \mathrm{Na}^{+}, \mathrm{Mg}^{2+}$, que nos da indicios de las rocas donde se encuentran los mantos acuíferos o por donde circula el agua.

\subsection{REACCIONES PRINCIPALES DEL HIERRO}

El hierro (II) sulfato (VI) presente en las aguas no puede precipitarse dentro de las características físicoquímicas de las aguas del río Tarucachi pero eso lo hace en los terrenos agrícolas, por lo cual necesita del ión $\mathrm{Ca}^{2+}$ para flocular, como anteriormente se ha demostrado en el laboratorio (1), y luego precipitarse.

Los iones metálicos hidratados son ácidos en el sentido dado por BRONSTED. El equilibrio ácido-base para el hierro (III) es :

$$
\left[\mathrm{Fe}\left(\mathrm{H}_{2} \mathrm{O}\right)_{6}\right]^{3+}+\mathrm{H}_{2} \mathrm{O} \longrightarrow\left[\mathrm{Fe}\left(\mathrm{H}_{2} \mathrm{O}\right)_{5}(\mathrm{OH})\right]^{2+}+\mathrm{H}_{3} \mathrm{O}+
$$

Al transferir un protón a una molécula solvente de agua $\left(\mathrm{H}_{2} \mathrm{O}\right)$ coordinada, se convierte en hidronio (3). La base conjugada puede entonces transferir nuevamente un protón.

$\left[\mathrm{Fe}\left(\mathrm{H}_{2} \mathrm{O}\right)_{5}(\mathrm{OH})\right]^{2+}+\mathrm{H}_{2} \mathrm{O} \longrightarrow\left[\mathrm{Fe}\left(\mathrm{H}_{2} \mathrm{O}\right)_{4}(\mathrm{OH})_{2}\right]^{+}+\mathrm{H}_{3} \mathrm{O}^{+}$

Pueden continuar transferencias similares. El orden y magnitud de la primera constante de acidez del ion hierro (III) acuoso es aproximadamente igual al comportamiento del ácido fosfórico. De acuerdo al pH

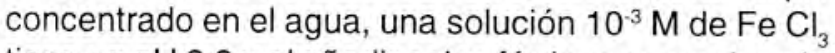
tiene un $\mathrm{pH} 3,3$ y al añadir sales férricas se produce la disminución en el pH y reducción de la alcalinidad.

Los complejos de hierro (II), hidróxidos, tienen una enorme tendencia a polimerizarse, produciendo muchas reacciones; la reacción más simple en las especies dímeras es:

$2\left[\mathrm{Fe}\left(\mathrm{H}_{2} \mathrm{O}\right)_{6}(\mathrm{OH})\right]^{2+} \longrightarrow\left[\mathrm{Fe}_{2}\left(\mathrm{H}_{2} \mathrm{O}\right)_{8}(\mathrm{OH})_{2}\right]^{4+}+2 \mathrm{H}_{2} \mathrm{O}$

Los dos iones metálicos se encuentran ligados probablemente mediante dos puentes hidroxo.

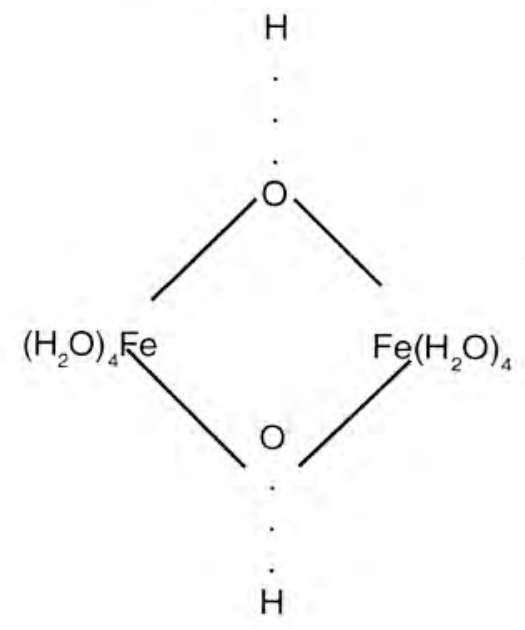


Este dímero sufre reacciones hidrolíticas adicionales y forma complejos mayores de hidróxidos, los cuales forman más puentes hidroxo; por ejemplo:

$\left[\mathrm{Fe}_{2}\left(\mathrm{H}_{2} \mathrm{O}\right)_{8}(\mathrm{OH})_{2}\right]^{4+}+\mathrm{H}_{2} \mathrm{O} \longrightarrow\left[\mathrm{Fe}_{2}\left(\mathrm{H}_{2}\left(\mathrm{H}_{2} \mathrm{O}\right)_{7}(\mathrm{OH})_{3}\right]^{3+}+\mathrm{H}_{3} \mathrm{O}^{+}\right.$

Una secuencia de las reacciones hidroliticas, acompañadas probablemente de reacciones de deshidratación, conduce a una coordinación progresiva del hierro (III) a través de enlaces con los grupos hidroxo, existiendo una mayor repulsión entre los iones y una mayor tendencia hacia la polimerización. Finalmente se forman de este modo, polímeros hidroxo coloidales y precipitados insolubles de hierro (III) óxido hidratados.

La asignación de una estructura polimérica al hierro (III) óxido hidratado se basa en la observación del momento magnético del óxido menor que el de las sales de Fe (III).

Debido a que los complejos catiónicos hidroxo hierro (III) prevalecen en soluciones ácidas o neutras, se forman coloides de hierro (III) óxido, cargados positivamente en esta gama de $\mathrm{pH}$. Sin embargo la polimerización aniónica $\left[\mathrm{Fe}(\mathrm{OH})_{4}\right]$, produce coloides bajo condiciones alcalinas.

TABLA No. 1: Características del agua del río Tarucachi.

\begin{tabular}{|c|c|c|}
\hline PARÁMETRO & & MÉTODO \\
\hline $\begin{array}{l}\text { Aspecto } \\
\text { Turbidez } \\
\mathrm{pH} \\
\text { Alcalinidad a la } \\
\text { Fenoltaleina } \\
\text { Alcalinidad Total } \\
\mathrm{CO}_{2} \text { libre } \\
\text { Temperatura }\end{array}$ & $\begin{array}{l}\text { con grumos rojizos } \\
2,3-3,0 \text { UNT } \\
2,35 \cdot 3,45 \\
0 \\
0 \\
182 \mathrm{ppm} \\
11,8 \cdot 18,2\end{array}$ & $\begin{array}{l}\text { Óptico } \\
\text { Nefelometria } \\
\text { Conductimetria }\end{array}$ \\
\hline
\end{tabular}

Fuente : Lab. de físico-química, UNJBG.

\subsection{COAGULACIÓN DE HIERRO (III)}

Los complejos hidroxo polinucleares disueltos, intermedios en la transición de los iones de hierro a óxido hidratados, son coagulantes eficientes. Bajo condiciones favorables de $\mathrm{pH}$, temperatura y tiempo de añejamiento, los productos de hidrólisis de iones metálicos poseen una mayor carga que la de los iones metálicos mismos y se absorben más fuertemente sobre
TABLA No. 2: ANÁLISIS QUÍMICO DEL AGUA

\begin{tabular}{|c|c|c|}
\hline SUSTANCIA & $\begin{array}{c}\text { CONCENTRACIÓN } \\
{\mathrm{Mg} / \mathrm{dm}^{3}}^{3}\end{array}$ & MÉTODO \\
\hline $\mathrm{Na}$ (K incluido) & $86,73-100,15$ & Cálculo \\
\hline $\mathrm{Ca}$ & $168-416$ & EDTA \\
\hline $\mathrm{Mg}$ & $28,8-32,6$ & EDTA \\
\hline $\mathrm{Fe}$ (Total) & $3,25-4,5$ & Ortofenantrolina \\
\hline Fe soluble & $0,53-0,8$ & Ortofenantrolina \\
\hline $\mathrm{Cu}$ & $0,45-0,70$ & \\
\hline $\mathrm{Cl}$ & $20,000-21,000$ & $\begin{array}{l}\mathrm{Hg}_{2} \mathrm{Gl}_{2} \\
\text { difenilcarbozona }\end{array}$ \\
\hline $\mathrm{SO}_{4}{ }^{2}$ & 539 & Cálculo \\
\hline As & $0,055-0,123$ & $\mathrm{Ag}$ DDTC \\
\hline
\end{tabular}

FUENTE : Lab. de físico-química, $U N J B G$

dispersiones coloidales que sobre iones metálicos no ionizados. De acuerdo a la bibliografía, se pueden deducir las siguientes conclusiones:

a) $\mathrm{A} \mathrm{pH}$ menor de tres, el Fe (III) no se encuentra hidrolizado, pero a $\mathrm{pH}$ cuatro, todo el hierro es trivalente.

b) A valores más altos de $\mathrm{pH}$, los complejos hidroxo polinucleares altamente cargados se forman y se adhieren fuertemente sobre la superficie de los coloides, capaces de invertir la carga de los coloides.

c) A partir de la concentración de inversión de carga, seconcluye que las especies hidrolizadas y no $\mathrm{el} \mathrm{Fe}^{3+}$ libre causan la inversión de carga.

d) A concentraciones más elevadas de Fe (III) se precipita hierro (III) óxido.

Las relaciones de solubilidad y el $\mathrm{pH}$ de los iones $\mathrm{Fe}$ (II) con los otros estados de oxidación más elevados los convertirá en solubles. En presencia del oxígeno disuelto el Fe (II) es termodinámicamente inestable a todos los $\mathrm{pH}$ de las aguas naturales.

$$
\begin{aligned}
& 2 \mathrm{Fe}_{2}^{+}+1 / 2 \mathrm{O}_{2}+5 \mathrm{H}_{2} \mathrm{O} \longrightarrow 2 \mathrm{Fe}(\mathrm{OH})_{3}+4 \mathrm{H}^{+} \\
& \mathrm{K}=5,8\left(25^{\circ} \mathrm{C}\right)
\end{aligned}
$$

Las reacciones de oxigenación van acompañadas de una reducción en el $\mathrm{pH}$.

\subsection{REACCIONES DEL CALCIO CARBONATO (IV)}

La mayor parte de cationes multivalentes en el agua se pueden precipitar en cantidades predecibles como carbonatos o hidróxidos. 
Esto se ha demostrado en el laboratorio, es decir, que estos compuestos gobiernan la solubilidad, la estabilidad y precipitación que inician el $\mathrm{Ca}^{2+}$ y $\mathrm{Mg}^{2+}$, sin embargo, no basta conocer la solubilidad (Tabla No. 3) porque cuando se agrega o entra en contacto el agua ácida del río Tarucachi con la $\mathrm{Ca} \mathrm{CO}_{3}$ (caliza), las especies que se forman son:

$$
\mathrm{Ca} \mathrm{CO}_{3}+\mathrm{H}_{2} \mathrm{O} \longrightarrow \mathrm{Ca}(\mathrm{OH})_{2}+\mathrm{CO}_{2}
$$

y se disuelven en el agua como $\mathrm{Ca}^{2+}$ y $\mathrm{CO}_{3}{ }^{2-}$ en una base y los productos de su reacción con el agua son predominantemente $\mathrm{HCO}_{3}$ y $\mathrm{OH}$

En términos generales el $\mathrm{Ca} \mathrm{CO}_{3}$ reaccióna:

$$
\mathrm{Ca} \mathrm{CO}_{3(} \mathrm{S}_{3}+\mathrm{H}^{+}<\longrightarrow \mathrm{Ca}^{2+}{ }_{(a \mathrm{~g})}+\mathrm{HCO}_{3(\mathrm{ag})}
$$

Es obvio que al disminuir el $\mathrm{pH}$, la solubilidad del $\mathrm{CaCO}_{3}$ (s) aumenta, mientras que al subir, ésta disminuye.

La evaluación cuantitativa de las relaciones del sistema que determinan las concentraciones de equilibrio (Tabla No. 3) de una solución está sujeta a un tratamiento exacto y sistemático sin suposiciones apriori.

El programa elaborado para el río Tarucachi comprende :

a) Enumeración de todas las especies que existieran en la solución en equilibrio (Tabla No. 2). b) Establecimiento de los diversos equilibrios de los iones principales para realizar la remoción de hierro y arsénico con $\mathrm{Ca} \mathrm{CO}_{3}$.

c) Selección de las constantes de equilibrio que identifican a las constantes de equilibrio de los componentes del soluto en las aguas del canal experimental.

En las aguas que tienen contacto con $\mathrm{Ca} \mathrm{CO}_{3}$, el pH es sobre todo una función de las cantidades relativas de $\mathrm{CO}_{2},\left(\mathrm{HCO}_{3}\right)$ y $\mathrm{CO}_{3}^{2}$.

Si se conocen dos de ellas, el $\mathrm{pH}$ se calcula fácilmente por medio de las relaciones de la ley de acción de masas y las constantes de equilibrio apropiadas. Las solubilidades de los carbonatos $\mathrm{Fe} \mathrm{CO}_{3}$ y Ca $\mathrm{CO}_{3}$ están siempre interrelacionadas. Los cálculos del equilibrio se han aplicado mediante:

a) Las concentraciones de equilibrio de toda clase de solutos en una suspensión de $\mathrm{Ca} \mathrm{CO}_{3}$ en agua pura (destilada) no abierta a la atmósfera.

b) En él se calculó las concentraciones de equilibrio de los solutos del $\mathrm{Ca} \mathrm{CO}_{3}$ con variación del $\mathrm{pH}$ y del tiempo en aguas del rio Tarucachi, encontrándose que el $\mathrm{pH}$ se elevaba en función del tiempo de contacto del agua con la caliza, en aproximadamente tres minutos a $\mathrm{pH}=6,8$.

\begin{tabular}{|c|c|c|c|c|c|}
\hline \multirow{2}{*}{ REACCIÓN } & \multirow{2}{*}{ K } & \multicolumn{4}{|c|}{ TEMPERATURA $^{\circ} \mathrm{C}$} \\
\hline & & 5 & 10 & 15 & 20 \\
\hline $\mathrm{CO}_{2}(\mathrm{~g})+\mathrm{H}_{2} \mathrm{O}(\mathrm{c}) \rightarrow \mathrm{H}_{2} \mathrm{CO}_{3}$ & $\mathrm{~K}$ & 1,20 & 1,20 & 1,34 & 1,41 \\
\hline $\mathrm{H}_{2} \mathrm{CO}_{3} \rightarrow \mathrm{HCO}_{3}+\mathrm{H}^{+}$ & $k_{1}$ & 6,52 & 6,52 & 6,42 & 6,38 \\
\hline $\mathrm{HCO}_{3} \rightarrow \mathrm{CO}_{3}^{2-}+\mathrm{H}^{+}$ & $k_{2}$ & 10,56 & 10,56 & 10,43 & 10,38 \\
\hline $\mathrm{CaCO}_{3}(\mathrm{~s}) \rightarrow \mathrm{Ca}^{2+}+\mathrm{CO}_{3}^{2-}$ & $k_{5}$ & 8,09 & 8,09 & 8,22 & 8,28 \\
\hline $\mathrm{CaCO}_{3}(\mathrm{~s})+\mathrm{H}^{+} \rightarrow \mathrm{Ca}^{2+}+\mathrm{HCO}_{3}$ & $\mathrm{Ks} / \mathrm{K}_{2}$ & $-2,47$ & $-2,34$ & $-2,21$ & $-1,99$ \\
\hline $\mathrm{CaCO}_{3}(\mathrm{~s}) \rightarrow \mathrm{H}_{2} \mathrm{CO}_{3}+2 \mathrm{HCO}_{3}^{-}$ & $\frac{\mathrm{Ks} / \mathrm{K}_{1}}{\mathrm{~K}}$ & 4,05 & 4,12 & 4,21 & 4,36 \\
\hline $\mathrm{H}_{2} \mathrm{O} \rightarrow \mathrm{H}^{+}+\mathrm{OH}^{-}$ & $\mathrm{KW}$ & 14,731 & 14,54 & 14,35 & 14,00 \\
\hline
\end{tabular}

TABLA No. 3 Solubilidad del $\mathrm{CA} \mathrm{CO}_{3}$ y Constante de Equilibrio de Carbonatos. 
c) El canal proyectado es un sistema abierto a la atmósfera, en este caso no se puede despreciar el $\mathrm{CO}_{2}$ de la atmósfera, sino tratar al $\mathrm{H}_{2} \mathrm{CO}_{3}$ como ácido no volátil.

\subsection{REACCIONES DE CALCIO Y HIERRO EN AGUAS DEL RÍO TARUCACHI}

El hierro (II) sulfato (VI) presente en las aguas del rio Tarucachi no puede precipitarse solo, necesita del $\mathrm{Ca}^{2+}$ para flocular y luego precipitarse, considerando la composición química del río y de la caliza. Además de las reacciones anteriores tenemos:

$\mathrm{Fe} \mathrm{SO}_{4}+\mathrm{Ca}\left(\mathrm{HCO}_{3}\right)_{2}<\longrightarrow \mathrm{Fe}\left(\mathrm{HCO}_{3}\right)_{2}+\mathrm{Ca} \mathrm{SO} \mathrm{S}_{4}$

y como se seguirán formando iones $\mathrm{Ca}^{2+}$ se producirá $\mathrm{Ca}(\mathrm{OH})_{2}$, entonces:

$$
\mathrm{Fe}\left(\mathrm{HCO}_{3}\right)_{2}+2 \mathrm{Ca}(\mathrm{OH})_{2} \longleftrightarrow \mathrm{Fe}(\mathrm{OH})_{2}+2 \mathrm{Ca} \mathrm{CO}_{3}
$$

El hierro (II) hidróxido es oxidado a hierro (III) hidróxido por el oxígeno disuelto en el agua del canal, por el flujo turbulento.

$$
\left.4 \mathrm{Fe}(\mathrm{OH})_{2}+\mathrm{O}_{2}+\mathrm{H}_{2}\right) \longleftrightarrow-\longrightarrow \mathrm{Fe}(\mathrm{OH})_{3}
$$

El hierro (III) hidróxido insoluble se forma como un flóculo gelatinoso; pero cuando existe hierro (III) sulfato (VI) para formar el glomérulo no necesita oxígeno del aire.

Transformado el hierro en $\mathrm{Fe}^{3+}$ se convierte en medio de floculación al reaccionar con el ion hidrógeno carbonato (IV), formando partículas hidróxido coloidal cargadas positivamente.

$$
\mathrm{Fe}^{3+}+3 \mathrm{HCO}_{3} \longrightarrow \mathrm{Fe}(\mathrm{OH})_{3}+3 \mathrm{CO} 2
$$

Estas partículas de hidróxido absorben partículas suspendidas en el agua y se sedimentan al alcanzar cierto tamaño de esta manera se puede eliminar particulas de $\mathrm{Fe}^{3+}$ en suspensión en el agua. La desferrización ideal debe ser menor de $0,15 \mathrm{mg}$.

\section{III.CANAL EXPERIMENTAL}

\subsection{ANTECEDENTES.}

De acuerdo al Proyecto de Investigación "Interacción y Eliminación de Arsénico y Hierro del rio Tarucachi" realizado por el Msc. Leoncio Molina, bajo convenio Universidad de Surrey (Reino Unido) y Universidad Nacional de San Agustín de Arequipa (Perú) y financiado por el Consejo Británico y el CONCYTEC, se determinó que con la caliza y en condiciones más óptimas con calcita, ambos $\mathrm{Ca} \mathrm{CO}_{3}$, es posible elevar el $\mathrm{pH}$ de las aguas del río Tarucachi que se observa en la Tabla No. 1 y llevarlas a su pH 6,8, que la hace apta para la agricultura y uso pecuario; eliminando así la contaminación de hierro, arsénico y la acidez.

\subsection{CARACTERÍSTICAS DEL CANAL EXPERIMENTAL}

\subsubsection{Ubicación y Características del Canal Experimental}

Éste se construyó en el cauce del canal que viene de la quebrada KEJANE con la carretera Tacna-Tarata y el partidor Vila Vila de la comunidad de Estiquepampa con las siguientes características geométricas.

$$
\begin{array}{lrr}
\text { - Base } & : & 0,8 \mathrm{~m} . \\
\text { - Altura } & : & 0,3 \mathrm{~m} . \\
\text { - Longitud del canal } & : & 75,0 \mathrm{~m} .
\end{array}
$$

El material de construcción fue piedra caliza $\left(\mathrm{CaCO}_{3}\right)$ $y$ arcilla (Fig No. 1 y No. 2)

La piedra caliza fue acarreada de la cantera de PALQUILLA, teniendo un contenido de $\mathrm{CaCO}_{3}$ puro del $40-60 \%$, la pendiente fue del $2 \%$ y el caudal del canal es aproximadamente $90 \mathrm{l} / \mathrm{seg}$.

\subsubsection{Características de los Filtros}

a) Caracteristicas del filtro mayor

- Profundidad : $1,0 \mathrm{~m}$

- Pendiente : $12,0 \%$

- Longitud de filtro : $8,0 \mathrm{~m}$

- Forma de la base: romboidal doble (Ver fig. No. 3)

b) Características del filtro menor (por construirse)

- Profundidad : $0,5 \mathrm{~m}$

- Pendiente : $6,0 \%$

- Longitud del filtro : 4,0 m

- Forma de la base: romboidal doble 
CANAL EXPERIMENTAL SECCIÓN DE CANAL

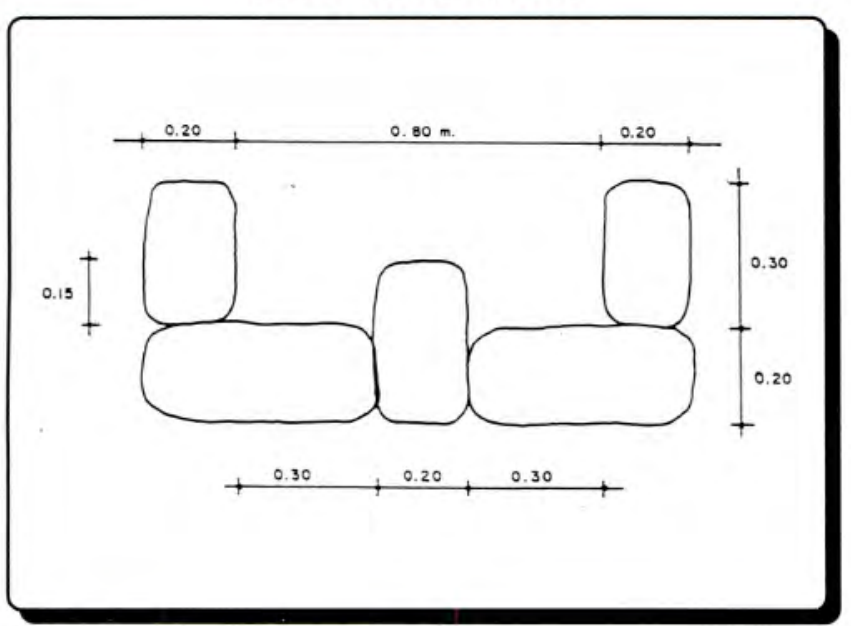

Fig. $N^{o} 1$
CANAL EXPERIMENTAL

VISTA DE PLANTA

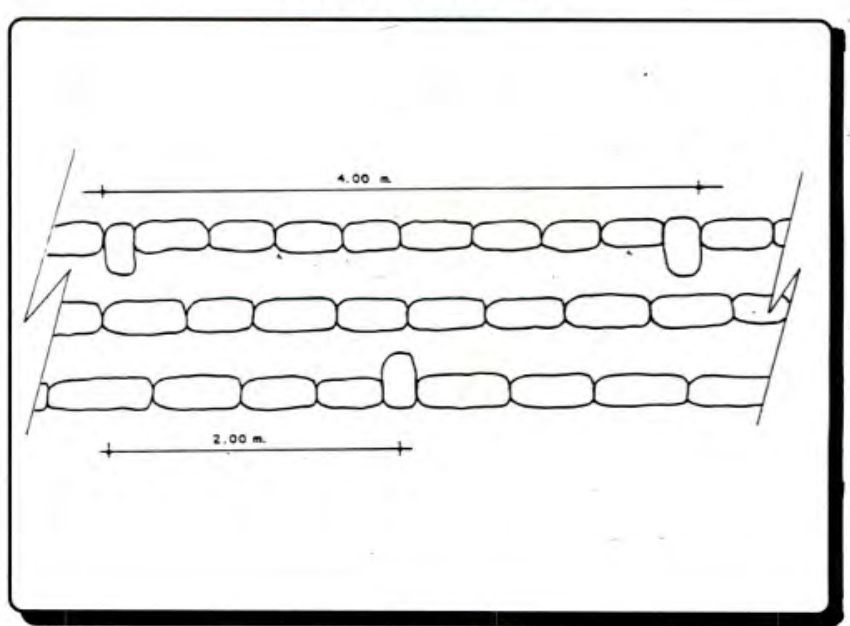

Fig. $N^{o} 2$

FILTRO PRINCIPAL DEL CANAL VISTA DE PLANTA
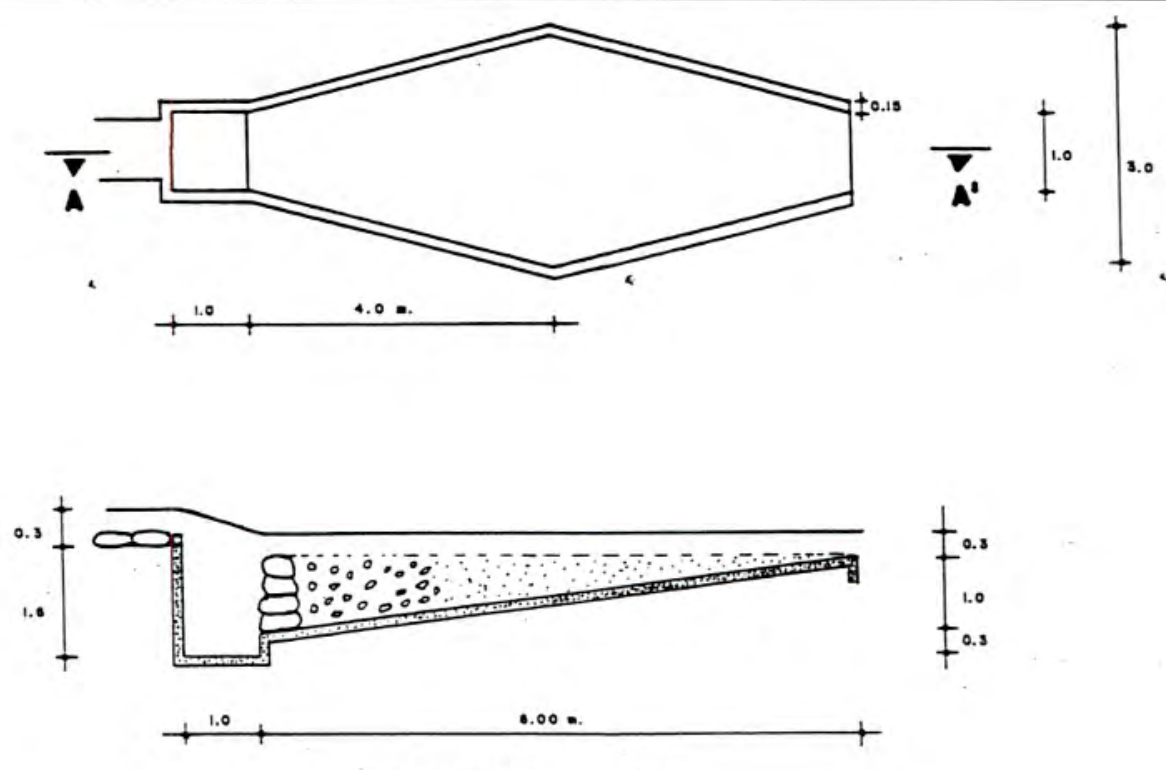

SEccion $A-A^{\prime}$

c) Material usado en los filtros

- Piedra caliza

: $\phi$ promed. $60 \mathrm{~cm}$.

- Grava

$: \phi$ de $3 \mathrm{~cm}$.

- Arena gruesa

d) Puntos de control (ver Anexo No. 2)

- Antes del ingreso al canal experimental punto 1
Fig. $N^{o} 3$

- Antes de ingreso al filtro mayor punto. 2

- Antes de ingreso al filtro menor punto 3

- Después de salir del filtro menor.

3.3. CONSTRUCCIONES PARA DETERMINAR LAS CARACTERÍSTICAS DEL CANAL. 


\subsubsection{La pendiente}

El arrastre del agua fluyente en el canal experimental, es análogo a la fricción ejercida por un cuerpo sobre un plano inclinado, esta fricción física incrementada por la reacción química de la caliza $\left(\mathrm{CaCO}_{3}\right)$ con el agua ácida es la que se considera.

Debido a que el volumen de agua en contacto con la superficie unitaria $\left(\mathrm{m}^{2}\right)$ del canal es igual al radio hidráulico

$$
\mathrm{R}=\tau \delta
$$

donde :

$\delta=$ Intensidad de la fuerza de tracción.

$\tau=$ Peso específico del agua a la temperatura promedio.

$R=$ Radio hidráulico de la sección llana.

= Pendiente del fondo o pérdida de carga en una longitud unitaria del canal, cuando el flujo es constante y la superficie del agua es paralela al fondo del canal.

Se ha calculado el $2 \%$ de pendiente del canal experimental.

\subsubsection{Flotación}

Las partículas en el canal, resultantes de la reacción de la caliza con las burbujas del $\mathrm{CO}_{2}$ que se adhieren a ellas, proporcionan fuerza ascendente al conjunto de partículas. Las burbujas de $\mathrm{CO}_{2}$ (gas) y de oxígeno disuelto son tales, que se observa a la partículas subiendo a la superficie por empuje hidrostático (lupa).

En el caso del canal experimental, las burbujas de gas se forman por la reacción de la caliza a la presión atmosférica de Tarata, en este caso la presencia de sales coloidales de hierro crean una estructura reticular que permite atrapar con mayor facilidad las burbujas de $\mathrm{CO}_{2}$.

El rendimiento de un sistema de flotación con $\mathrm{CO}_{2}$ depende de la relación entre $\mathrm{K}(\mathrm{g})$ de $\mathrm{CO}_{2}$ y los sólidos en flotación. La relación entre el cociente $\mathrm{G} / \mathrm{S}$ y la solubilidad del $\mathrm{CO}_{2}$, la presión de trabajo y la concentración de sólidos.

En el canal experimental se crea un sistema de tres fases (gas-líquido-sólido):
a) Tensión gas-sólido.
b) Tensión sólido-líquido
c) Tensión gas-líquido.

En conjunto determinan la magnitud del ángulo de contacto en el punto de conjunción de las tres fases, mientras mayor es este ángulo, mayor es la tendencia de las burbujas a adherirse a las partículas y éstas permanezcan en suspensión.

\subsubsection{Floculación}

Producto de la reacción química de la caliza, iones de hierro en $\mathrm{pH}$ ácido se forman complejos de $\mathrm{Fe}$ y $\mathrm{Ca}$, como se aprecia ópticamente en las muestras; esta reacción favorecida por el flujo turbulento producto del diseño del canal con "aletas" transversales de caliza, tal como se aprecia en la Fig. No. 2, sirven para la rotación de la masa del líquido y activar el mezclado de los iones:

\section{Fig. № 04 VELOCIDAD DE FORMACIÓN DE GLOMERULOS}

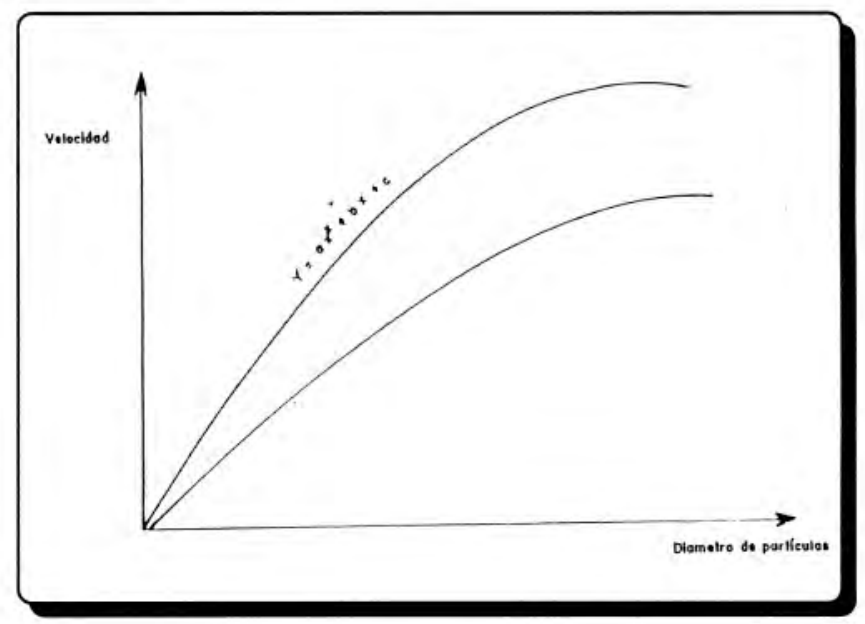

$$
\mathrm{HCO}_{3}, \mathrm{CO}_{3}{ }^{2-}, \mathrm{Ca}^{2+}, \mathrm{Fe}^{2+}, \mathrm{Fe}^{3+}, \mathrm{SO}_{4}{ }^{2-}, \mathrm{OH}^{+}, \mathrm{H}^{+} \text {, etc. }
$$

Un mayor contacto del líquido con la caliza, además favorece las reacciones:

$$
\begin{array}{ll}
\mathrm{Ca}^{2+}+\mathrm{H}_{2} \mathrm{SO}_{4} & \longrightarrow \mathrm{Ca} \mathrm{SO}_{4}+2 \mathrm{H}^{+} \\
\mathrm{Ca}(\mathrm{OH})_{2} & \longrightarrow \\
\mathrm{OH}+\mathrm{H}^{+} & \left.\longrightarrow \mathrm{Ca}^{2+}+2 \mathrm{OH}-\right] \\
& \longrightarrow \mathrm{H}_{2} \mathrm{O}
\end{array}
$$

Uno de los problemas que se presenta es el crecimiento de los flóculos hasta tamaños que producen su sedimentación, por esto es importante cuidar la velocidad y la turbulencia del agua para romper los flóculos en partículas más pequeñas.

\subsection{VELOCIDAD DE ARRASTRE}

La velocidad de arrastre es importante en la operación de evitar la sedimentación. En el canal 
experimental debe mantenerse la velocidad lo suficientemente elevada a fin de que las partículas sólidas no se depositen.

La velocidad crítica viene dada por la ecuación de campo, utilizando los estudios de Shields.

$1 / 2$

$\mathrm{VH}=\underline{8 \mathrm{~K}(\mathrm{~S}-1) \mathrm{gd}}$

$f$

Donde :

$\mathrm{VH}=$ Velocidad horizontal para producir el arrastre.

$\mathrm{S}=$ Peso específico de los flóculos de $\mathrm{Ca} \mathrm{SO}_{4} \mathrm{y}$ complejos de hierro.

$\mathrm{d}=$ Diámetro de las partículas.

$\mathrm{K}=$ Constante que depende del tipo de materia que arrastra.

En las tablas, $\mathrm{K}=0,06$ es el valor típico para materia floculante y entremezclada como los flóculos f es el factor de rozamiento de Darcy Weisbach que depende de las características de la rugosidad del canal $f=0,03$; $\mathrm{f} y \mathrm{k}$ con adimensionales.

\subsection{LA SEDIMENTACIÓN}

La sedimentación es reducida por las corrientes entre las que se encuentran :

1) Corrientes turbulentas creadas en el canal.

2) Corrientes superficiales producidas por el viento.

3) Corrientes organizadas por la diferente densidāa del agua fría de la parte inferior y el agua calentada en la superficie o viceversa.

La cuantía de la floculación en el canal depende de las oportunidades de contacto que tengan los iones de $\mathrm{SO}_{4}{ }^{2-}$ con la caliza, variando ésta con la superficie de contacto, velocidad, $\mathrm{pH}$, etc.

La sedimentación depende de muchas variables dentro las cuales consideraremos:

$\mathrm{F}=$ Fuerza total de resistencia.

$\delta=$ Densidad

$\mathrm{D}=$ Diámetro de la partícula

$\mathrm{V}=$ Viscosidad

$A=$ Area del sólido en contacto con el fluido.

$Q^{\prime}=$ Determinado experimentalmente

$U=$ Velocidad del agua debajo del sólido

Cuando la fuerza de resistencia $F$ es igual a la fuerza de la gravedad alcanza su valor límite:

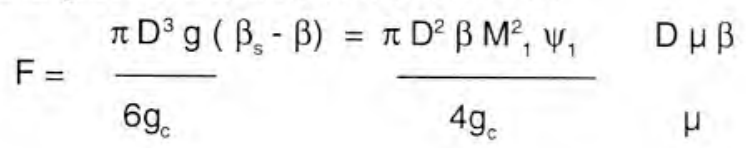

Fig. № 5 Sedimentación de Partículas.

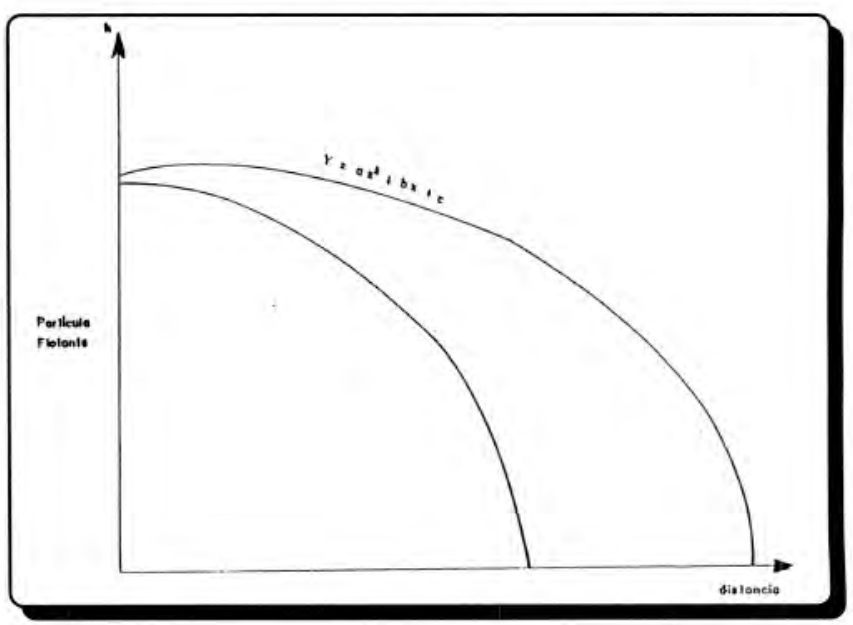

Cuando el movimiento de la partícula se encuentra en flujo turbulento

$$
F=\frac{\pi D^{2} \beta^{2} M^{2} C}{8 g_{c}}
$$

$\mathrm{C}=$ Coeficiente de arrastre y es análogo al factor de fricción.

En el caso del canal experimental $\mathrm{C}=0,20-0,44$ como una buena aproximación.

La sedimentación depende de la turbulencia en la corriente del agua, la rugosidad de la superficie del canal y del tamaño del fóculo, densidad, etc. , y de la acción del $\mathrm{CO}_{2}$ y $\mathrm{O}^{2}$ como elementos de flotación de las partículas y complejos en suspensión.

\subsection{RESISTENCIA AL MOVIMIENTO DE PARTÍCULAS}

Las partículas se mueven en forma compleja por las formas geométricas diversas, por esta razón las fórmulas son de aplicación limitada.

$$
\begin{aligned}
& \text { error }^{-}= \pm 20 \% \text { en } \mathrm{C}_{\text {vs }} \mathrm{R}_{\mathrm{c}} \\
& \mathrm{Rc} \_ \text {_ }
\end{aligned}
$$

La velocidad de sedimentación es de 40-60\% de las partículas esféricas, según la bibliografía para las condiciones dadas. La velocidad límite de sedimentación de las esferas de cuarzo (arena) a 20 ${ }^{\circ} \mathrm{C}$ en función del diámetro de la partícula para la zona 
es de 0,01 a 1,00 mm; la densidad de la caliza 2,7 g $\mathrm{cm}^{-3}$ (Daten sammlung chemie in SI) viscosidad del agua a $15^{\circ} \mathrm{C}$ es 1,1404 centi poise $=0.01\left(\mathrm{~g}\right.$-masa $\left.\mathrm{cm}^{3}\right)$

Para flujo laminar, según la Ley de Stockes

$$
\mu_{15}=D^{2}(2,70-1,00)(981)=9,000 D^{2}
$$

(18) $(0,01)$

Puesto que $D \mu_{15}, s / \mu$ en este caso debe ser menor que 0,1 , sustituyendo $\mu_{15}$

$$
D \mu_{15}=\frac{(9,000)(1,00) D^{3}}{(0,010)}=9\left(10^{5} D^{3)}<0,1\right.
$$

$D^{3}<0,111 \times 10^{-6} \mathrm{~cm}^{3}$

$D<0,0048 \mathrm{~cm}$

$D<0,048 \mathrm{~mm}$

La Ley de Stocks se aplica para esferas con diámetros menores que $0,048 \mathrm{~mm}$ y se puede calcular la velocidad límite de sedimentación (gráfico 14-16) (4).
$\mathrm{D}(\mu \mathrm{m})$
$\left(\mu \mathrm{mms}^{-1}\right)$
0,02
0,36
0,10
9,00

Para la región de régimen completamente turbulento: 1,000 - $R_{c}-200,000$ se supondrá que el coeficiente de arrastre es igual a 0,44.

Según la ecuación :

$$
\mu_{15}=\left[\frac{4 D_{c}\left(\beta_{t}-\beta_{o}\right) g}{3}\right]^{0.5}
$$

$\mu_{15}=\left[\frac{(4((2,70-1,000)(981) D}{(3)(0,44)(1,00)}\right]^{0.5}$

$\mu_{15}=70 D^{0,5}$

Para $R_{c}>1,0000$

$$
D \mu_{t} \beta_{\mu}=\frac{70,0 D^{1,5}}{0,01}=7,0000 D^{1,5} \geq 1,0000
$$

$D^{15}>0,1428$

$D \geq 0,274 \mathrm{~cm}=2,74 \mathrm{~mm}$

El máximo valor de $D$ corresponde a $R_{c}=200,000$ mucho mayor que cualquier partícula que se considere en el gráfico correspondiente.

$$
\mathrm{D}(\mathrm{mm}) \quad \mu(\mathrm{mm} / \mathrm{s})
$$

$\begin{array}{rr}20 & 990 \\ 3 & 384\end{array}$

Para el número de Reynolds comprendido entre 0,1 y 1,0000 se tiene:

$$
\begin{aligned}
& \mu_{15}=46,5(D / C)^{0,5} \\
& R_{c}=500 \mathrm{c}=0,55 \\
& \mu_{15}=62,6 D^{0,5} \mathrm{y} \mathrm{D}^{1,5}=0,0798 \mathrm{~cm}^{1,5} \\
& D=0,185 \mathrm{~cm} \mu=27 \mathrm{~cm} / \mathrm{s}
\end{aligned}
$$

Para el proceso de clasificación hidráulica se aplica en el canal experimental la distancia de 4 metros entre las aletas" de caliza que se han construido en el canal experimental.

\subsection{MEZCLADOR DE SALTO HIDRÁULICO}

El vertedero construido en el canal es un salto hidráulico hacia el aliviadero donde el flujo entrante se expande. La pérdida de carga es apreciable, pero mucha de la potencia asociada no se dispersa en forma útil. El periodo de retención es breve y sirve como mezclador instantáneo para poder pasar al filtrado.

\subsection{FILTRACIÓN}

La teoría de la filtración es un campo en el que hay un gran desarrollo matemático, pero la aplicación de esta teoría a los problemas prácticos es incompleta. Esto se debe a que es muy difícil precisar el tamaño de la partícula a filtrar como a la composición y diámetros de la piedra caliza y arena gruesa de los filtros. 
El tipo de filtros a constituirse en el canal experimental es abierto, de filtración rápida y tiene caliza y arena, tal como se especifica en 2.2.2., porque este tipo permite manejar volúmenes de líquido grandes con el mínimo costo y remover el hierro.

\subsection{REMOCIÓN DE LAS IMPUREZAS SEDIMENTADAS}

Ésta comienza y se realiza primero en toda la longitud del canal para limpiar la superficie de la caliza y continuar de esta forma la reacción química con el agua.

Esto se hizo usando ramas de arbustos de las zonas

TABLA No. 4

pH DE AGUAS EN EL CANAL EXPERIMENTAL (12 DÍAS DESPUES)

\begin{tabular}{|l|l|l|}
\hline $\mathbf{M}$ & $\mathbf{p H}$ & $\mathbf{T}^{\circ} \mathbf{C}$ \\
\hline 0 & 2,35 & 14,5 \\
10 & 3,0 & 14,5 \\
20 & 3,2 & 15,0 \\
20 & 3,3, & 14,5 \\
40 & 3,4 & 14,5 \\
50 & 3,5 & 14,5 \\
60 & 3,6 & 14,5 \\
70 & 3,7 & 14,5 \\
\hline
\end{tabular}

FUENTE : Recolección de datos experimentales.

TABLA No. 5

pH DE AGUAS EN EL CANAL EXPERIMENTAL ( 30 DÍAS DESPUES)

\begin{tabular}{|c|c|c|}
\hline $\mathbf{M}$ & $\mathbf{p H}$ & $\mathbf{T}^{\circ} \mathbf{C}$ \\
\hline 0 & 2,5 & 17 \\
10 & 2,8 & 17 \\
20 & 3,0 & 17 \\
30 & 3,3 & 17 \\
40 & 3,5 & 17 \\
50 & 3,6 & 17 \\
60 & 3,7 & 17 \\
70 & 3,8 & 17 \\
\hline
\end{tabular}

FUENTE : Recolección de datos experimentales. aledañas al canal experimental y escobillones domésticos.

En los filtros, los sedimentos deben limpiarse utilizando palanas, luego de desviarse el curso del canal, para de esta forma evitar cortar el agua a usarse en el riego.

En cada instalación de filtros, debe existir como mínimo dos. Cuando un filtro está fuera de funcionamiento por limpieza, la otra unidad debe ser capaz de proveer el servicio requerido en el ritmo de filtración.

TABLA No. 6 pH DEL AGUA EN EL CANAL EXPERIMENTAL (75 DIAAS DESPUÉS)

\begin{tabular}{|r|l|l|}
\hline $\mathbf{M}$ & $\mathbf{p H}$ & $\mathbf{T}^{\circ} \mathbf{C}$ \\
\hline 0 & 2,5 & 17 \\
10 & 2,7 & 17 \\
20 & 3,0 & 17 \\
30 & 3,1 & 17 \\
40 & 3,2 & 17,5 \\
50 & 3,3 & 18,0 \\
60 & 3,35 & 18,0 \\
70 & 3,4 & 18,2 \\
\hline
\end{tabular}

FUENTE : Recolección de datos experimentales.

TABLA No. 7 CORRECCION DEL PH POR T ${ }^{\circ}$ EN AGUAS DEL RÍO TARUCACHI

\begin{tabular}{|c|c|c|c|l|}
\hline $\mathbf{T}^{\circ} \mathbf{C}$ & $\mathbf{p H}$ & $\mathbf{p H}$ & $\mathbf{p H}$ & $\mathbf{p H}$ Prom \\
\hline 0 & 3,35 & 3,38 & 3,36 & 3,36 \\
4 & 3,30 & 3,29 & 3,29 & 3,29 \\
8 & 3,28 & 3,28 & 3,26 & 3,27 \\
10 & 3,26 & 3,27 & 3,26 & 3,26 \\
18 & 3,19 & 3,20 & 3,21 & 3,20 \\
22 & 3,17 & 3,16 & 3,18 & 3,17 \\
25 & 3,15 & 3,13 & 3,13 & 3,13 \\
30 & 3,12 & 3,11 & 3,11 & 3,11 \\
40 & 3,09 & 3,09 & 3,09 & 3,09 \\
\hline
\end{tabular}

FUENTE : Recolección de datos experimentales laboratorio. 
TABLA No. 8

pH EN EL AGUA DEL CANAL EXPERIMENTAL

(PROMEDIO)

\begin{tabular}{|l|l|l|}
\hline Distancia $\mathbf{M}$ & $\mathbf{p H}$ & $\mathbf{T}^{\circ} \mathbf{C}$ \\
\hline 0 & 2,48 & 15,0 \\
10 & 2,77 & 15,0 \\
20 & 3,02 & 15,0 \\
30 & 3,22 & 15,0 \\
40 & 3,38 & 15,0 \\
50 & 3,49 & 15,0 \\
60 & 3,56 & 15,0 \\
70 & 3,58 & 15,0 \\
80 & 3,55 & 15,0 \\
90 & 3,48 & 15,0 \\
100 & 3,36 & 15,0 \\
\hline
\end{tabular}

FUENTE : Datos experimentales

\section{IV.RESULTADOS EXPERIMENTALES}

\subsection{IMPORTANCIA DEL $\mathrm{pH}$}

Los cambios del $\mathrm{pH}$ difieren dentro de la naturaleza ácida de la solución iónica y están determinados por las magnitudes relativas de $\mathrm{H}^{+}$y $\mathrm{OH}$ en término de parámetros simples.

En general, la ecuación de las aguas del río Tarucachi, que corresponde a la suma de varias contribuciones químicas, está dada por el producto de las ecuaciones o constantes de equilibrio para las ecuaciones químicas del hierro, arsénico, $\mathrm{Ca}^{2+}, \mathrm{CO}_{2}$, $\mathrm{SO}_{4}{ }^{2-}$, etc.
Los datos experimentales obtenidos se ven en las Tablas Nos 4, 5, 6, 7 y los promedios se determinan en la curva que tiene la forma parabólica $Y=a x^{2}+b x+c$

Con los datos de la Tabla No. 7 elaboramos el Gráfico No. 1 mediante el cual calculamos el incremento del $\mathrm{pH}$ en el canal experimental $\mathrm{pH}_{1}$

$$
\begin{aligned}
& \mathrm{pH}_{1}=3,58-2,47 \\
& \mathrm{pH}_{1}=1,11
\end{aligned}
$$

Los procesos físico-químicos acuosos en hidraúlica tienen un error de $\pm 20 \%$, aplicando este dato al incremento del $\mathrm{pH}, \mathrm{pH}_{2}$ :

$$
\begin{aligned}
& \mathrm{pH}_{2}=(1,11)(0,80) \\
& \mathrm{pH}_{2}=0,888 \\
& \mathrm{pH} \text { Calculado }=3,364 \text { (experimental) }
\end{aligned}
$$

En el gráfico No. 1 la función queda determinada para el cálculo del $\mathrm{pH}$ por :

$$
\mathrm{pH}=-0,00023214 \mathrm{~m}^{2}+0,032043 \mathrm{~m}+2,476389
$$

El pH máximo se encuentra a 3,58 a una longitud del canal de :

$$
\mathrm{m}: 70
$$

Aplicamos el porcentaje de error por exceso para calcular la distancia del canal y obtener el incremento del $\mathrm{pH}$ de 0,888 tenemos :

$$
\begin{aligned}
& \mathrm{d} \text { Calculado }=(70 \mathrm{~m})(1,20) \\
& \mathrm{d} \text { Calculado }=84 \mathrm{~m}
\end{aligned}
$$

La presencia del hierro soluble o insoluble, así como las partículas de $\mathrm{Ca} \mathrm{SO}_{4}$, deben ser eliminadas del flujo del canal, para ello usamos los filtros. Éstos se ubicarán de acuerdo a la distancia calculada con error por deficiencia :

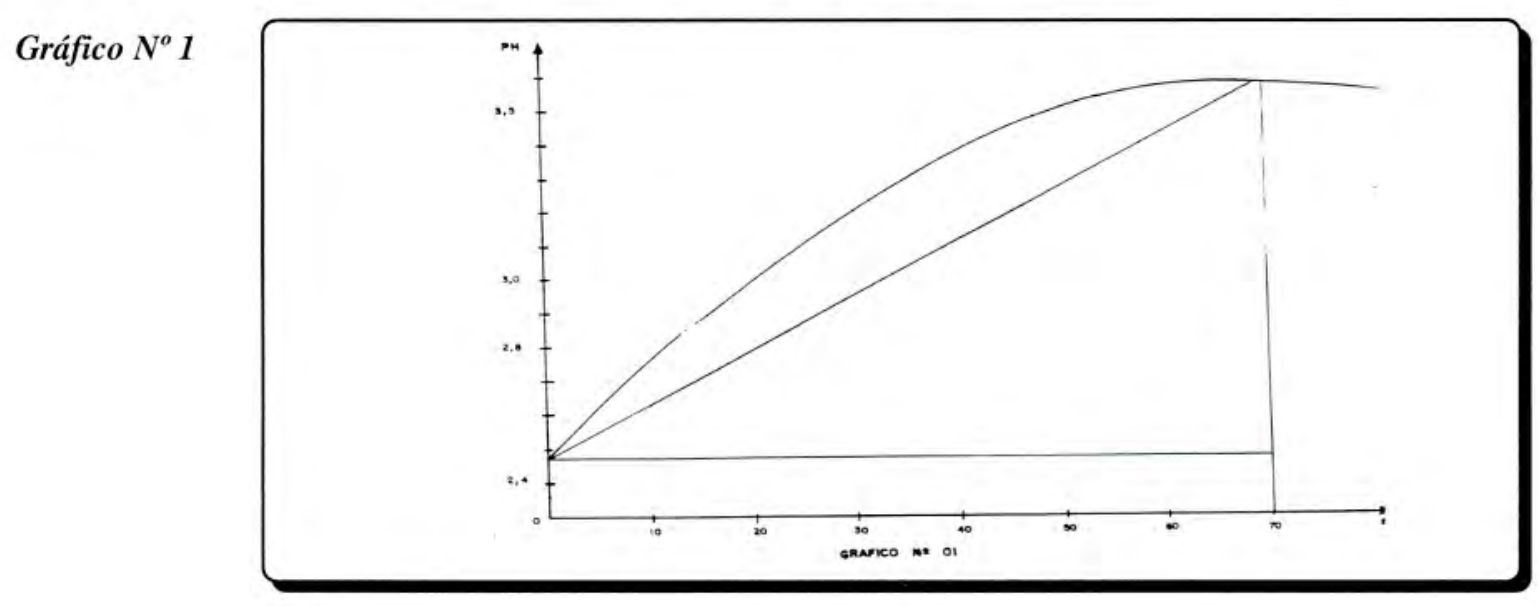




$$
\begin{aligned}
\mathrm{d} \text { filtros } & =(70 \mathrm{~m})(0,8) \\
& =56 \sim 60 \mathrm{~m}
\end{aligned}
$$

Con esta información determinamos el incremento del $\mathrm{pH}$ para el tratamiento con piedra caliza $\left(\mathrm{Ca} \mathrm{CO}_{3}\right)$ desde el carácter ácido promedio que tiene el agua del río Tarucachi hasta alcanzar el $\mathrm{pH}$ neutro que habilitaría las aguas para el uso agropecuario normal, $\mathrm{pH} 3$.

$$
\begin{aligned}
& \mathrm{pH}_{3}=7,0-2,47 \\
& \mathrm{pH}_{3}=4,53
\end{aligned}
$$

Hallamos el factor para deteriminar la distancia del canal experimental :

$$
\begin{aligned}
& \mathrm{pH}_{3}=4,53=5 \\
& \mathrm{pH}_{1} \quad 0,888
\end{aligned}
$$

La distancia calculada del canal experimental de acuerdo a los datos experimentales :
(d) canal $=(84 \mathrm{~m})(5)$
(d) canal $=420 \mathrm{~m}$

Con la distancia del canal experimental calculada, encontraremos el número de filtros a construirse, de acuerdo al diseño anteriormente especificado :

No. de filtros $=$

$$
420
$$

$$
=7^{60}
$$

que ahora consideraremos como corrección para construir el canal experimental en segunda aproximación.

\subsection{HIERRO EN EL AGUA TRATADA}

El hierro se muestra en la Tabla No. 8. Del análisis vemos que existe una tendencia a disminuir el hierro, esto mejoraria ostensiblemente cuando se construyan los filtros, y esta disminución contribuiría notoriamente a la elevación del $\mathrm{pH}$; porque los polímeros hidroxocoloidales precipitados, insolubles de óxidos de hierro hidratado quedarían atrapados.

\subsection{ARSÉNICO EN AGUA DEL CANAL EXPERIMENTAL}

El hierro que se encuentra poco hidrolizado, al tratarlo con la caliza $\left(\mathrm{Ca} \mathrm{CO}_{3}\right)$, forma redes con puentes hidroxo mencionados en 1.2. ; los dímeros y polímeros forman redes que atrapan al arsénico y otros iones haciéndolos precipitar y de esta forma se eliminará este elemento. Logrando la variación de la concentración del arsénico con, el pH-en el proyecto de investigación "Interacción y Eliminación de Arsénico y Hierro en Aguas del Río Tarucachi".

\subsection{CALCIO EN EL CANAL EXPERIMENTAL}

En la Tabla No. 8 apreciamos que el calcio total se incrementa significativamente, indicando que la reacción entre el agua y el $\mathrm{Ca} \mathrm{CO}_{3}$ (caliza) se realiza, indudablemente, faltando determinar el desgaste de la caliza para saber el tamaño a usarse en el emboquillado de los canales a construirse y el periodo de vida media.

\subsection{ESTABILIZACIÓN QUÍMICA}

El principio de solubilidad del $\mathrm{Fe} \mathrm{CO}_{3}$ tiene dos órdenes de magnitud menor que la solubilidad del $\mathrm{CaCO}_{3}$.

Dentro del análisis experimental, la estabilización química consiste en un ajuste del $\mathrm{pH}$ en el equilibrio con $\mathrm{CaCO}_{3}$, ya que el carácter ácido removerá el $\mathrm{CaCO}_{3}$ de la caliza.

Las condiciones de equilibrio anteriormente mencionadas quedan definidas por las ecuaciones:

$$
\begin{gathered}
\mathrm{CaCO}_{3}+\mathrm{H}^{+} \longrightarrow \mathrm{Ca}^{2+}+\mathrm{HCO}_{3}^{-} \\
\mathrm{K}=\left[\mathrm{Ca}^{2+}\right]\left[\mathrm{HCO}_{3}{ }^{-}\right]
\end{gathered}
$$

El punto de saturación del $\mathrm{CaCO}_{3}$ se caracteriza por:

$$
\left[\mathrm{H}^{+}\right]_{\text {eq }}=\left[\mathrm{H}^{+}\right] \text {agua río }
$$

Cuando $\left[\mathrm{H}^{+}\right]_{\text {agua }} \longrightarrow\left[\mathrm{H}^{+}\right]$equilibrio, el $\mathrm{Ca} \mathrm{CO}$ de la caliza se disuelve y esta solución proporcionará el $\mathrm{CO}_{2}$ al agua que ayudará la flotación de las partículas y coloides presentes en el agua.

Langelier ha determinado el índice de saturación :

$$
\mathrm{I}=\mathrm{pH}_{\text {agua }}-\mathrm{pH}_{\text {eq. }}
$$

En el caso de las aguas del río Tarucachi I es negativa, es corrosiva; porque el $\mathrm{pH}$ es ácido

\subsection{PH PROYECTADO}

Como se aprecia, los incrementos del $\mathrm{pH}$ van decreciendo, esto se debe a que la acción corrosiva de la $\left[\mathrm{H}^{+}\right]$disminuye al incrementarse el $\mathrm{pH}$, producto de la neutralización de los iones $\mathrm{Ca}^{2+}$ y de la eliminación del hierro. 


\subsection{LIMPIEZA DE LA CALIZA}

En los experimentos no se ha notado mayor diferencia del pH cuando se ha hecho la medición de las calizas con sedimentos en la superficie y cuando se ha hecho la limpieza de ellas. A pesar de esto, se recomienda la limpieza de las superficies de las piedras calizas cada mes, usando escobillones o ramas de arbusto.

TABLA No. 8

\begin{tabular}{|l|l|l|l|l|}
\hline No. & MUESTRA & FECHA & $\begin{array}{l}\text { Fc TOTAL } \\
\mathbf{m g} / \mathbf{d m}^{2}\end{array}$ & $\begin{array}{l}\mathbf{C a}^{2+} \\
\mathbf{m g} / \mathbf{d m}\end{array}$ \\
\hline 1 & Inicial & & 4,45 & 168 \\
2 & Final & & 4,3 & 277 \\
3 & Inicial & & 4,65 & 162 \\
4 & Intermedio & & 3,75 & \\
5 & Final & & 3,55 & 262 \\
6 & Inicial & & 4,05 & 170 \\
7 & Final & & 3,85 & 209 \\
8 & Inicial & & 3,65 & 165 \\
9 & Final & & 3,01 & 230 \\
\hline
\end{tabular}

FUENTE : Determinación experimental.

TABLA No. 9: VARIACIÓN CALCULADA DEL pH

\begin{tabular}{|l|l|l|l|}
\hline $\begin{array}{c}\text { ETAPA } \\
\text { CALCULADA }\end{array}$ & $\mathbf{p H}$ & $\mathbf{p H}$ & $\mathbf{p H}$ \\
\hline & & & \\
1 & 2,47 & 0,888 & 3,358 \\
2 & 3,358 & 0,808 & 4,166 \\
3 & 4,166 & 0,727 & 4,893 \\
4 & 4,893 & 0,646 & 5,539 \\
5 & 5,539 & 0,566 & 6,105 \\
6 & 6,105 & 0,486 & 6,591 \\
7 & 6,591 & 0,406 & 6,997 \\
\hline
\end{tabular}

FUENTE : Cálculo de los autores
ANEXO No. 33

VISCOSIDAD DEL AGUA

\begin{tabular}{|c|c|}
\hline $\mathrm{T}^{\circ} \mathrm{C}$ & CENTIPOISE \\
\hline 0 & 1,7921 \\
\hline 3 & 1,6191 \\
\hline 5 & 1,5188 \\
\hline 8 & 1,3860 \\
\hline 10 & 1,3077 \\
\hline 13 & 1,2028 \\
\hline 15 & 1,1404 \\
\hline 18 & 1,0359 \\
\hline 20 & $1,0005(1,0000)$ \\
\hline 23 & 0,9358 \\
\hline 25 & 0,8937 \\
\hline 28 & 0,8360 \\
\hline 30 & 0,8007 \\
\hline
\end{tabular}

FUENTE: Manual del Ing. Químico. J. Perry.

\section{IV.CONCLUSIÓN}

El canal experimental con emboquillado de caliza del 40 - $60 \%$ de $\mathrm{Ca} \mathrm{CO}_{3}$ para hacer apta el agua del río Tarucachi con el caudal de $90 \mathrm{l} . \mathrm{S}^{-1}$ que pasa por el partidor de Vila Vila tendrá $420 \mathrm{~m}$ de largo con las características reseñadas y debe tener siete (7) filtros. 


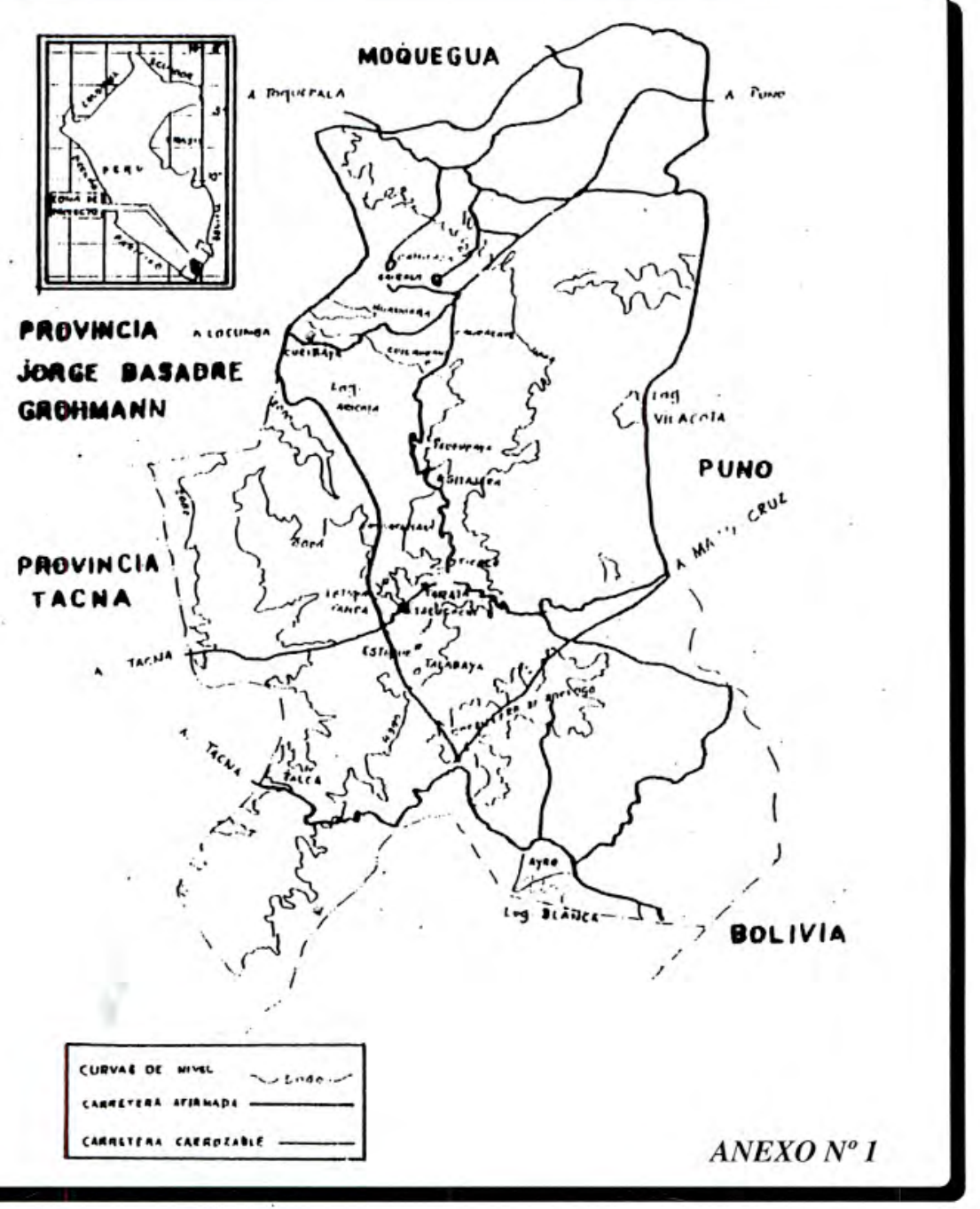

ANEXO № 2. CROQUIS : DISPOSICIÓN DE CANAL EXPERIMENTAL, FILTROS MAYOR Y MENOR 


\section{v. BIBLIOGRAFÍA}

NAMOR, Angela: "Comunicación Privada"

AMERICAN WATER WORKS ASSOCIATTION - AMERICAN PUBLIC HEALTH ASSOCIATION, "Standard Methodos for the Examinatione of water an waste water" 1970.

BRUCE Y WEISSEBERG, "Medical Progress" Vol. 291; №22

RAZMILIA, B.B. "Atomic Espectroscopy" Vol 7; No.1, Jan Feb, 1986.

GASTANY G. "Prospección y Explotación de las Aguas Subterráneas" Ed. Omega Año 1975.

FRIES - GESTROST, "Organic Reagents For Trace Analysis" Merck 1977.

DAGNINO, Orlando: "Técnicas Analíticas en la Determinación de Arsénico en aguas". Informe Chaco Argentina 1979.
PUNGOR; "Manual de laboratorio de Química"; BudapestHungría 1977.

IPCS: Arsenics, Envirenmental Helath Criterial 18, Finlandia, Año 1977.

WALKA, FIELD, SIMON: "Analysis and Removal Arsenic in Drinking Water" - Tesis University of SURREY, England 1982.

ALEXEIEV: "Semimicro Analisis Cualitativo" Ed. Limusa,1989.

FAIR, OKUN: "Purificación de Agua" Tomo II, Edit Limusa 1989.

"INTERNATIONAL STANDARS FOR DRINKING WATER" $3 a$. Edición of World Health Organization, 1971.

MOLINA, Leoncio: "Interacción y Eliminación de Arsénico y Hierro en agua del río Tarucachi. Tesis Mcs. Convenio Universidad San Agustín (Perú) - University of Surrey (England) Arequipa - Perú, 1989. 